\title{
Density effects on semi-dwarf and early flowering mutants of Arabidopsis thaliana under continuous light
}

\author{
Kei Takahashi, Miyuki Hara, Kana Miyata, Ryosuke Hayama, Tsuyoshi Mizoguchi* \\ Department of Natural Sciences, International Christian University (ICU), Osawa 3-10-2, Mitaka, Tokyo 181-8585, Japan \\ *E-mail: mtsuyoshi@icu.ac.jp Tel: +08-422-33-3247
}

Received July 1, 2016; accepted September 12, 2016 (Edited by T. Demura)

\begin{abstract}
Plant growth promotion and inhibition under low- and high-density conditions (referred to as the density effect) has been studied extensively. Here, we show that such density effects were unaffected by the position of wild-type (WT) and gibberellic acid insensitive ( $\mathrm{gai}$ ) strains of Arabidopsis thaliana (Arabidopsis) within pots. Additionally, petanko 1 (pta1) and pta5 were newly discovered alleles of the ROTUNDIFOLIA 3 (ROT3) and DWARF 4 (DWF4) genes that are involved in brassinosteroid biosynthesis. Unlike gai, the semi-dwarf mutants of pta1 and pta5 exhibited normal flowering times and a shortening of rosette leaves at high densities. Moreover, the pta1 and pta5 variants suppressed flowering stem shortening at high densities. pta5, but not pta1 suppressed the reduction in silique number at intermediate densities. SPINDLY (SPY) is a negative regulator of GA signaling, while PHYTOCHROME B (PHYB) is a red-light photoreceptor. High-density growth did not reduce the flowering time of $p h y B$ mutants, but did affect that of $s p y$ mutants. Neither spy nor phyB suppressed the shortening of rosette leaves at high densities; however, spy suppressed flowering stem shortening. Moreover, spy suppressed the reduction of silique number at high densities, while and phyB promoted the decrease. These data suggest that GA, BR, and light signaling pathways play important roles in the density effect.
\end{abstract}

Key words: Arabidopsis, density effects, flowering time, gibberellic acid, semi-dwarf.

The law of constant final yield (Kira et al. 1953; Pacala and Weiner 1991; Pearl and Parker 1922) states that the total biomass production of plants grown at different densities is constant following an initial period of growth (Weiner and Freckleton 2010). At high densities, leaf and stem lengths are shortened and the number of flower buds decreases. The flowering time is also accelerated under such conditions (Aihara 1968; Jennings and de Jesus 1968; Levin and Wilson 1978), and these phenomena are known as density effects (Fishman 1997; Pacala and Weiner 1991).

We recently established conditions to investigate density effects in Arabidopsis thaliana (Arabidopsis), as well as three additional Brassicaceae plants (Yamamoto et al. 2016). These plants exhibited short stems and leaves, and had accelerated flowering when grown at high densities. The acceleration of flowering at increased densities was suppressed by the gibberellic acid insensitive (gai) mutation (Koornneef et al. 1991; Peng et al. 1997) in the gibberellic acid (GA) pathway (Mutasa-Göttgens and Hedden 2009), but not by other late flowering mutations in the photoperiod/long-day pathway, including that of gigantea ( $g$ i Koornneef et al. 1991), late elongated hypocotyl; circadian clock associated 1 (lhy;cca1; Fujiwara et al. 2008; Mizoguchi et al. 2002), or in the flowering control locus A gene (fca; Koornneef et al. 1991) of the autonomous pathway. Since plant density affects plant growth, a detailed understanding of the molecular mechanisms surrounding such processes is important for plant science.

We hypothesize that the promotion of flowering by density effects is dependent upon the GA pathway; however, since gai strains are semi-dwarf mutants with short hypocotyls, leaf petioles, and blades, plant density may be lower than that of the wild-type (WT) when the same number of seeds are sown per pot. We previously discussed the density effects on growth and development, where ethylene, GA signaling, and flowering time genes are primary factors. Although several late flowering mutants were used, only the early flowering 3 mutant strain (elf3; Zagotta et al. 1996) was investigated previously (Yamamoto et al. 2016). Since then, many questions have been raised.

Density effects could be due to other plant hormones, including brassinosteroid (BR) and auxin (Busov et al. 2008; Hardtke et al. 2007). Additionally, the observed

Abbreviations: Arabidopsis, Arabidopsis thaliana; CCA1, CIRCADIAN CLOCK ASSOCIATED 1; ELF3, EARLY FLOWERING 3; GAI, GIBBERELLIC ACID INSENSITIVE; LHY, LATE ELONGATED HYPOCOTYL; LL, continuous light; PHYB, PHYTOCHROME B; PTA1, PETANKO 1; PTA5, PETANKO 5; SPY, SPINDLY.

This article can be found at http://www.jspcmb.jp/

Published online November 9, 2016 
dwarfism in extremely high-density conditions could be caused by the reduction of light quantity or changes in light quality. Thus, room for growth and development may be dependent on pot locations (e.g., the center or edge of pots).

Recently, we identified petanko 1 and 5 (pta1 and pta5; meaning flat in Japanese; Suzuki et al. 2016) as mutations that exaggerate the semi-dwarf phenotype of the lhy;ccal mutant (Fujiwara et al. 2008) under continuous light (LL). pta1 and pta5 are new alleles of the rotundifolia 3 (rot3; Kim et al. 1998) and dwarf 4 (dwf4; Choe et al. 1998) genes, respectively. Both ROT3/PTA1 and DWF4/PTA5 encode key enzymes involved in BR biosynthesis (Choe et al. 1998; Kim et al. 2005). The semi-dwarf phenotypes of pta1 and pta5 strains were less than that of gai.

GAI (Peng et al. 1997) positively regulates the GA signaling pathway, while SPINDLY (SPY, Jacobsen et al. 1996) negatively regulates the GA pathway. PHYTOCHROME B (PHYB) encodes a red-light photoreceptor (Reed et al. 1993) and cross-talk between the GA and light signaling pathways has been reported (Lor and Olszewski 2015). The gai mutation is dominantnegative, delays flowering time, and shortens hypocotyls, leaf blades and petioles, and flowering stems (Peng et al. 1997). By contrast, loss-of-function mutations in either the SPY or PHYB genes accelerates flowering and lengthens hypocotyls, leaf blades, and petioles (Jacobsen et al. 1996; Reed et al. 1993).

We first tested density effects on WT and gai plants (Figure 1) grown at either the center or edge of pots (Figure 2). WT and gai seeds were sown at densities of 5, $10,20,40$, and 60 per pot and grown under continuous light (LL). The number of cauline and rosette leaves (flowering time; Figure 2A-E), as well as the length of rosette leaves (Figure $2 \mathrm{~F}-\mathrm{J}$ ) and flowering stems (Figure $2 \mathrm{~K}-\mathrm{O}$ ), and the number of siliques (Figure 2P-T) from plants grown at the edge of pots were compared to those at the center of pots. As the density of WT plants per pot increased, the number of leaves and siliques decreased, while the length of rosette leaves and flowering stems shortened (Figures 1, 2A, 2B, 2E-G, 2J-L, 2O-Q, 2T).

As recently reported (Yamamoto et al. 2016), gai mutants exhibited lengthened flowering times at densities of 10 to 60 seeds per pot (Figures 1, 2C-E). Using a detailed statistical analysis, we found that gai mutants also had shortened leaf/stem lengths and a decreased number of siliques (Figures 1, 2H-J, 2O-Q, 2T). The percentages of the average length of $\mathrm{WT}$ rosette and cauline leaves grown at the edge and center of pots at a density of 60 were 65.3 and $63.2 \%$, respectively, compared to those at a density of 5 seeds per pot (Figure 2E). By contrast, the percentages of the average length of rosette and cauline leaves in the gai strain at the edge and center of pots were 82.1 and $82.4 \%$, respectively, at a density of 60 seeds per pot, compared to those at a density of 5 seeds per pot (Figure 2E). The percentages of the average length of WT rosette leaves grown at the edge and center of pots were 42.6 and $42.3 \%$, respectively, at a density of 60 seeds per pot, compared to those at a density of 5 seeds per pot (Figure 2J). In contrast, the percentages of the average length of rosette leaves of the gai strain grown at the edge and center of pots at a density of 60 seeds per pot were 60.8 and $62.6 \%$, respectively, compared to those at a density of 5 seeds per pot (Figure 2J). Notably, there was no statistical difference in flowering times and leaf lengths between WT and gai strains grown at the edge and center of pots (Student's $t$-test, $p>0.05$; Figure 2E, J).

The shortening of flowering stems was suppressed in the gai strain at densities of 10-40 seeds per pot (Figure $2 \mathrm{~K}-\mathrm{O}$ ), while the decrease in silique number was moderately suppressed in the gai strain at densities of 20 and 40 seeds per pot (Figure 2P-T). No significant difference in stem lengths or silique numbers was observed between WT and gai strains grown at the edge and center of pots (Student's $t$-test, $p>0.05$; Figure 2O, $\mathrm{T})$. Thus, the data suggested that the positions of plants in pots did not influence such density effects in WT and gai strains. Therefore, 10 plants were chosen randomly from the surface of pots and their leaf and stem lengths, as well as their silique counts were also assessed (Yamamoto et al. 2016).

In addition to the gai strain, we also assessed strains with semi-dwarf mutations in ROT3/PTA1 (Kim et al. 1998; Suzuki et al. 2016) and DWF4/PTA5 (Choe et al. 1998; Suzuki et al. 2016) (Figure 3). Compared to gai (Figure 3A-B, 3E), pta1 and pta5 strains had WT flowering times at high densities (Figure 3C-E). pta1 and pta5 did not suppress rosette leaf shortening at high densities (Figure $3 \mathrm{H}-\mathrm{J}$ ), while pta5 mutants had elevated rosette leaf shortening at densities of 20 and 40 seeds per pot (Figure 3I-J). pta1 and pta5 mutants had reduced flowering stem shortening at high densities (Figure 3M-O). Moreover, the significant differences between the stem lengths of WT and pta1 or pta5 were observed at densities of 10-60 seeds per pot (Student's $t$ test, $p<0.05)$. pta5, but not pta1, suppressed the decrease in silique number that was observed in $\mathrm{WT}$ at densities of 10 and 20 seeds per pot (Figure 3P-T). These data suggested that the BR and GA signaling pathways are involved in density effects. Although the density effect was partially suppressed in pta 1 and pta5, the phenotype was weaker than gai (Figure 3). The results suggest that GA has more important role in the density effect than BR.

We also investigated the density effects of mutations that cause early flowering and sensitivity to GA and light (i.e., spy and $p h y B$ ) using the elf3 strain (Zagotta et al. 1996) as the control (Figure 4). Higher seed densities 

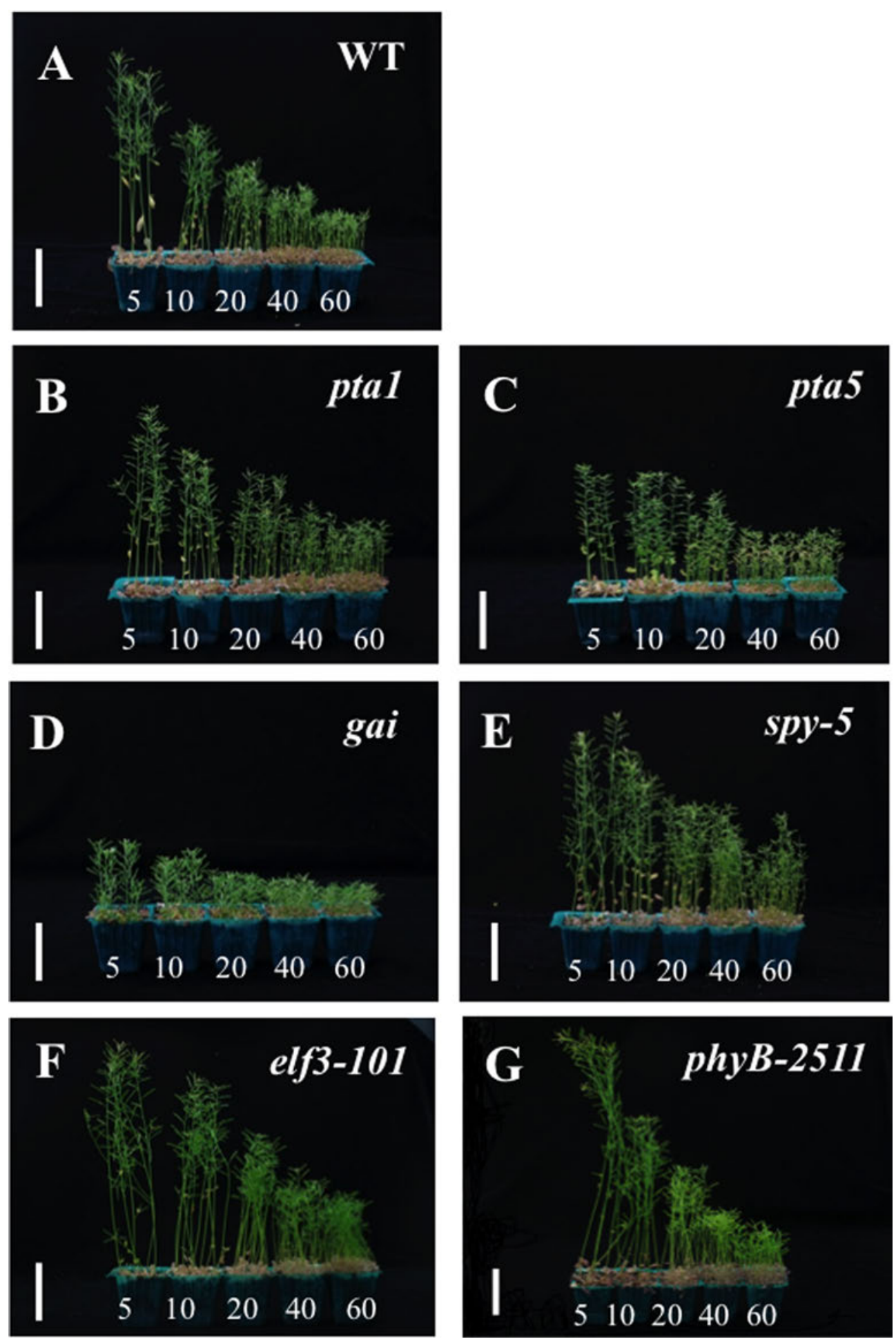

Figure 1. Density effects in Arabidopsis thaliana. Photograph of (A) WT (Ler), (B) pta1, (C) pta5, (D) gai, (E) spy-5, (F) elf3-101, and (G) phyB-2511. Seeds of Arabidopsis thaliana were sown at 5, 10, 20, 40, and 60 per pot, and grown at $24^{\circ} \mathrm{C}$ under continuous light (LL). Plants were photographed after bolting. Scale bars, $5 \mathrm{~cm}$.

did not accelerate flowering times in the phyB strain, but did in the spy strain (Figure 4A-E). The differences in the number of rosette and cauline leaves between WT and $s p y, p h y B$, or elf 3 were also significantly different at densities of 10-60 seeds per pot (Student's $t$-test, $p<0.05)$. spy and $p h y B$ did not suppress the shortening of the rosette leaves at high densities (Figure $4 \mathrm{~F}-\mathrm{J}$ ); however, spy suppressed the shortening of flowering stems at high densities (Figure $4 \mathrm{~K}-\mathrm{O}$ ). The difference between the stem length of WT and those of spy and elf3 mutants at densities of $10-40$ seeds per plot was statistically significant (Student's $t$-test, $p<0.05$ ). The 

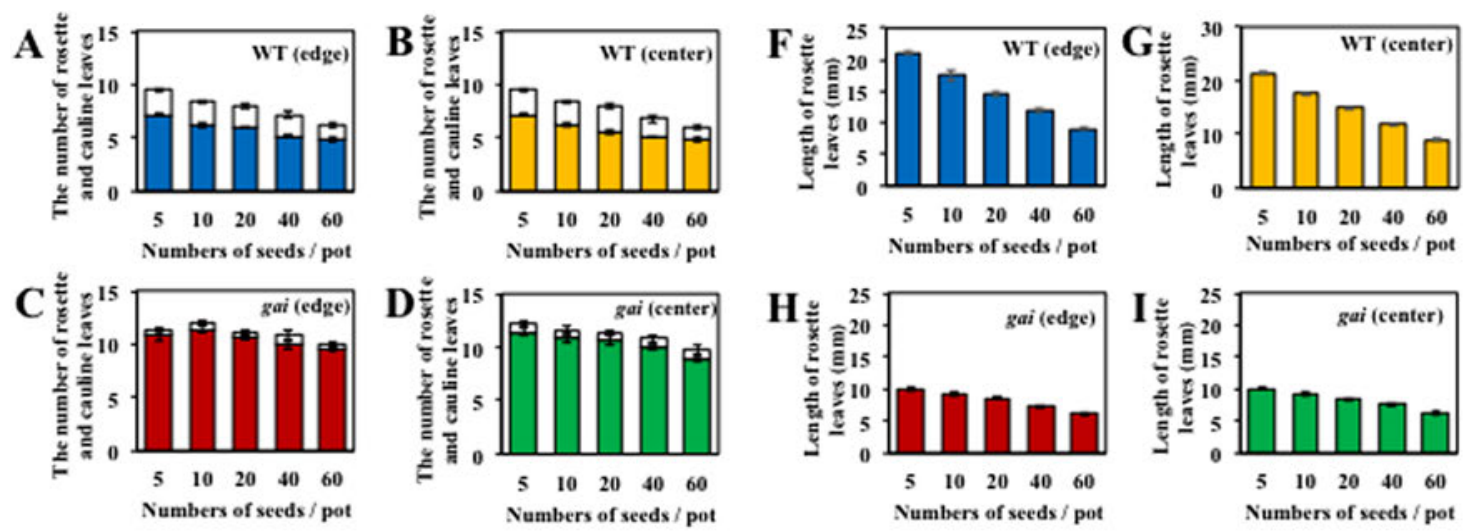

$\mathbf{E}$
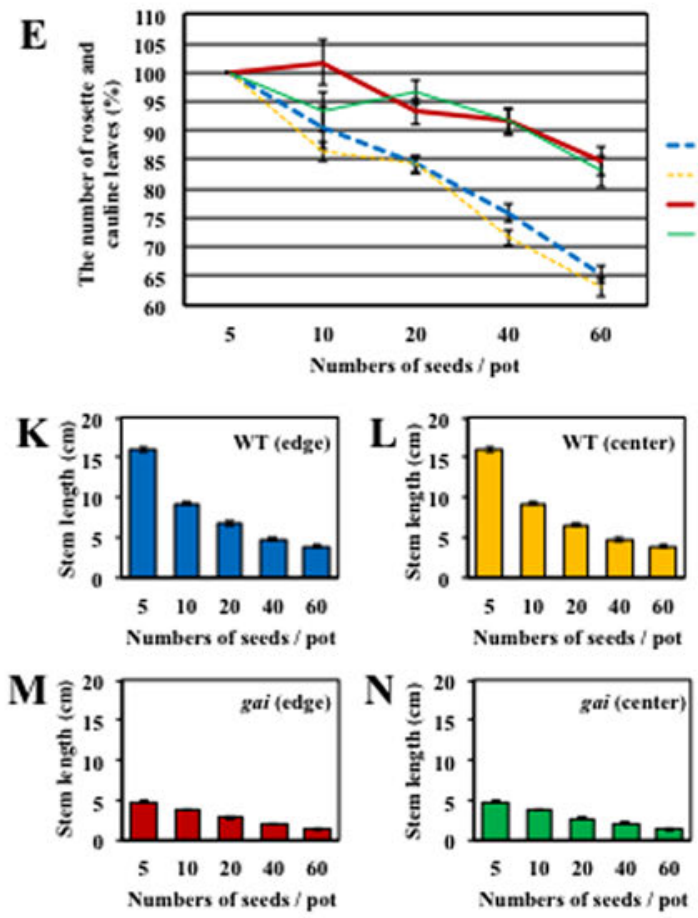

$\mathbf{O}$

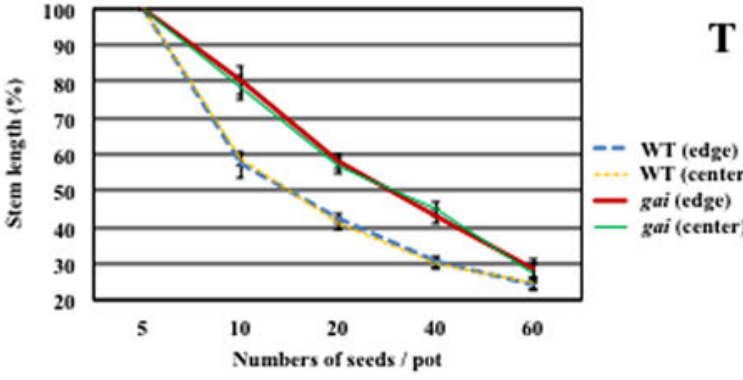

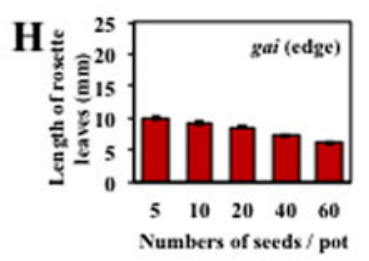

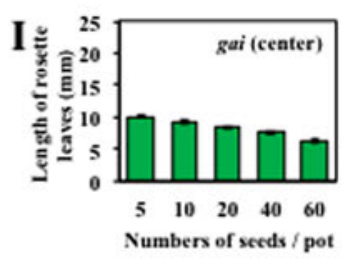

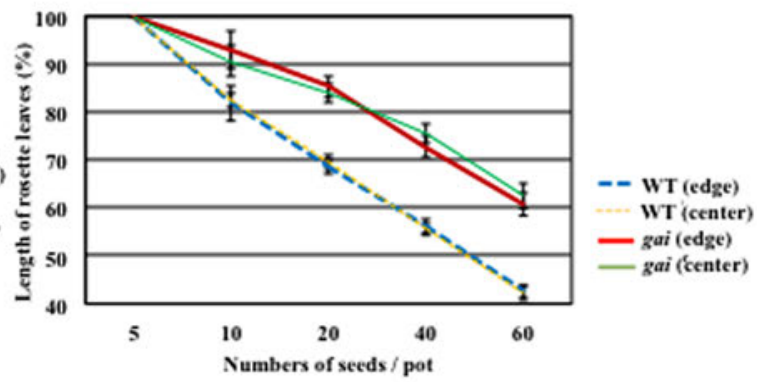
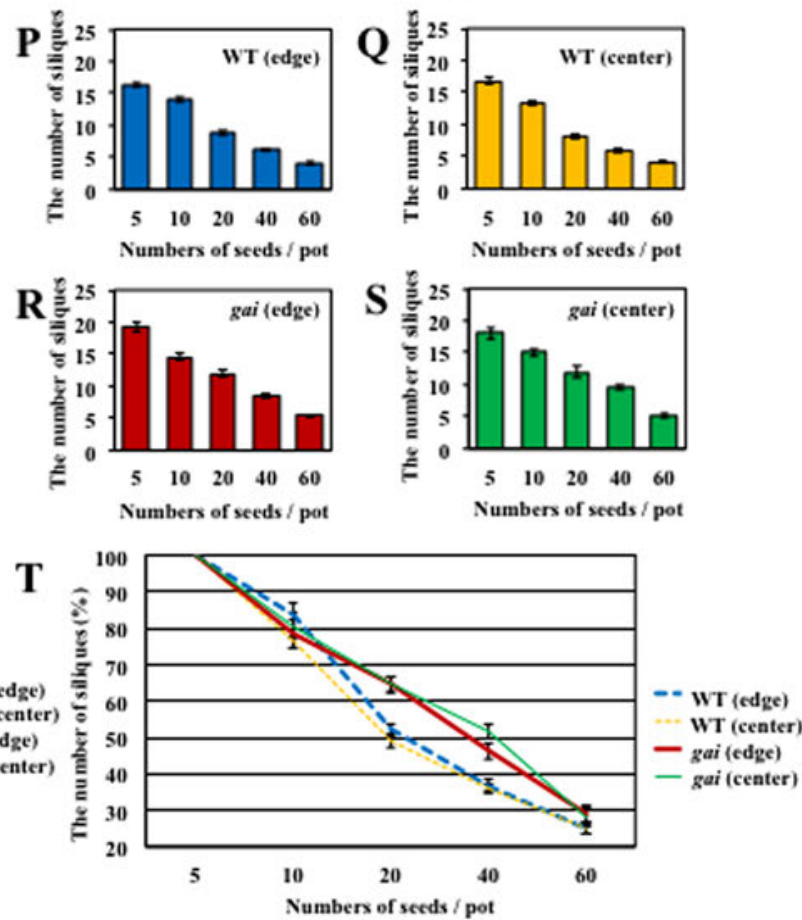

Figure 2. The effects of plant position in pots on density effects. Shown are measurements for WT plants grown at the edge (blue; A, F, K, P) and center (yellow; B, G, L, Q), and gai plants grown at the edge (red; C, H, M, R) and center (green; D, I, N, S) of pots. Decreases in rosette and cauline leaf number $(\mathrm{E})$, rosette leaf length $(\mathrm{J})$, flowering stem length $(\mathrm{O})$, and silique number $(\mathrm{T})$ relative to those at a density of 5 seeds per pot were measured. (A-E) Density effects of flowering times in WT (Ler) and gai strains. Rosette and cauline leaf numbers were determined after bolting. (F-J) Density effects on rosette leaf length in WT and gai strains. Rosette leaf lengths of the 3rd leaves were measured 3 weeks after sowing. (K-O) Density effects on flowering stem length in WT and gai strains. Flowering stem lengths were measured following bolting ( 6 weeks after sowing). (P-T) Density effects on silique number in WT and gai strains. Silique numbers were determined following bolting ( 6 weeks after sowing). Seeds $(5,10$, 20,40 , and 60 per pot) were grown at $24^{\circ} \mathrm{C}$ under LL. Data are presented as the means \pm SE $(n=5)$. No statistical difference was observed between the leaf numbers of WT and gai plants grown at the edge vs. the center of pots (Student's $t$-test, $p>0.05$ ).

decreases in stem length in the phyB mutant were enhanced at densities of 40 and 60 seeds per pot (Figure $4 \mathrm{O})$. The phyB mutant also decreased silique numbers at higher densities, while spy mutants did not (Figure 4P$\mathrm{T}$ ). Thus, GA and light signaling likely play key roles in density effects. 

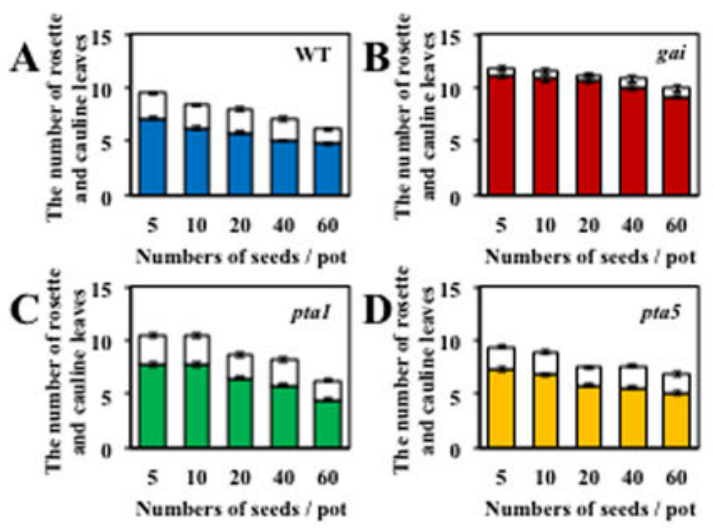

E
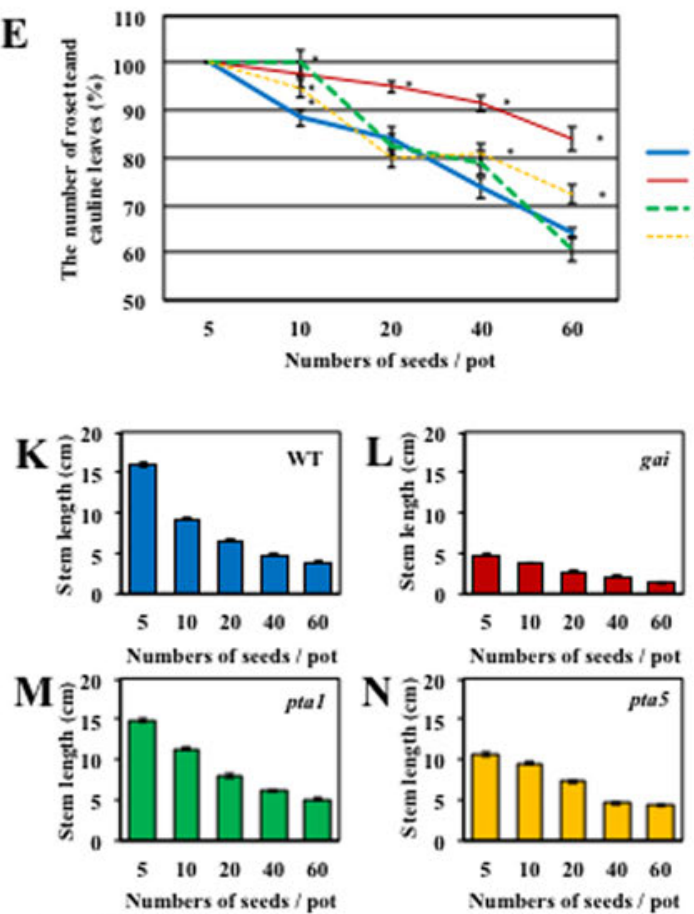

$\mathbf{O}$

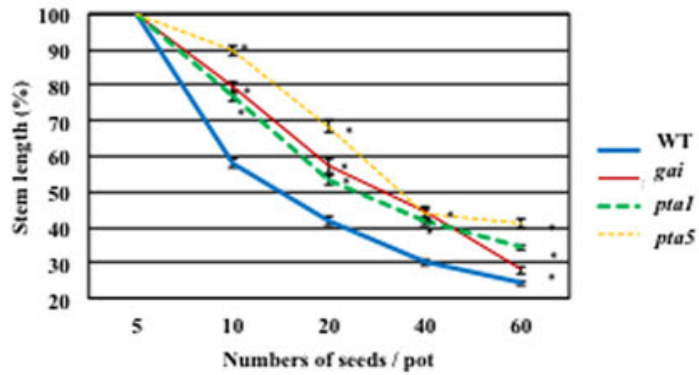

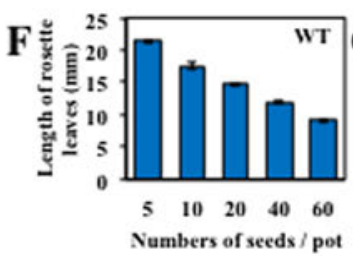
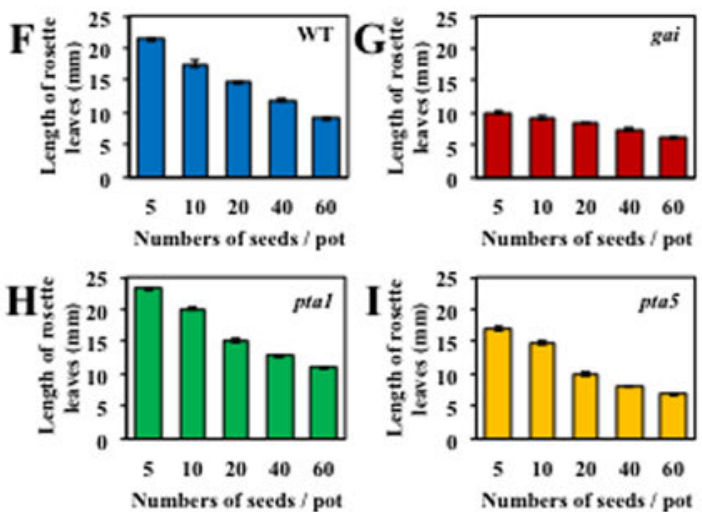

J
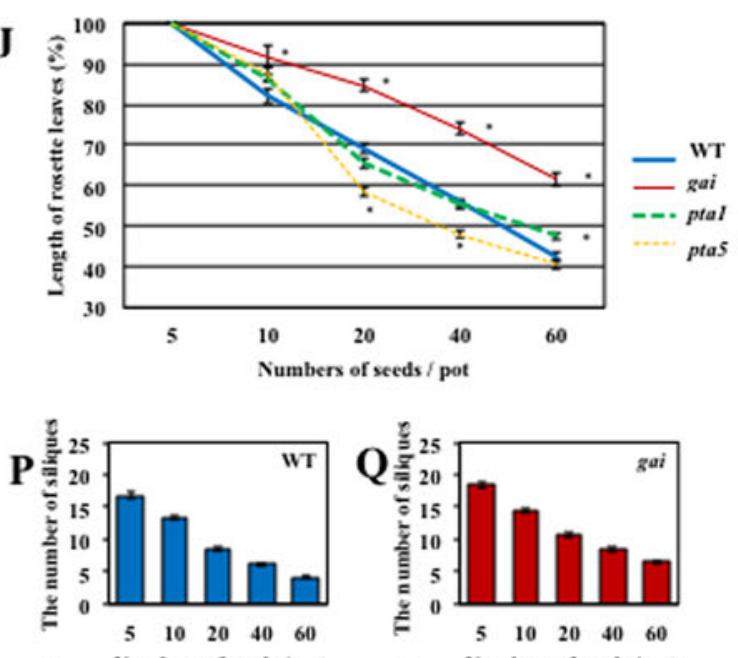

R 25 Numbers of seeds / pot

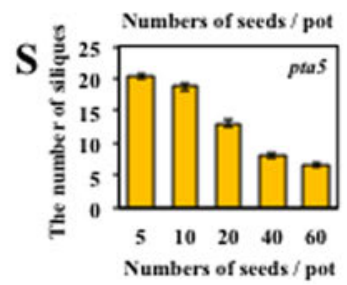

$\mathbf{T}$

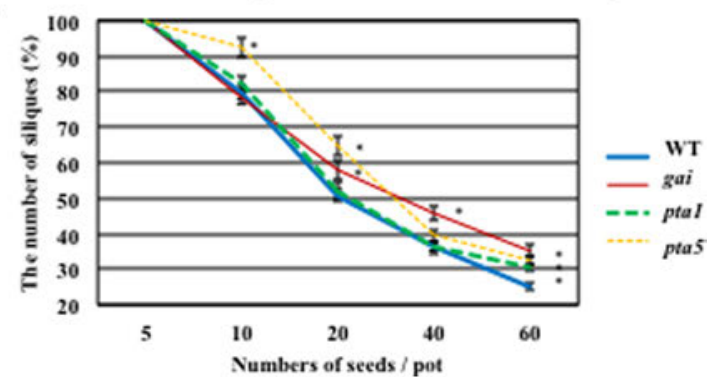

Figure 3. Density effects on flowering time, rosette leaf and flowering stem length, and silique number in gai, pta1, and pta5 mutants. Measurements are shown for WT (Ler, blue; A, F, K, P), gai (yellow; B, G, L, Q), pta1 (red; C, H, M, R), and pta5 (green; D, I, N, S) plants. Shown are the decreases in rosette and cauline leaf number $(\mathrm{E})$, rosette leaf length $(\mathrm{J})$, flowering stem length $(\mathrm{O})$, and silique number $(\mathrm{T})$ relative to those at densities of 5 seeds per pot. (A-E) Density effects on flowering time. Rosette and cauline leaf numbers were determined after bolting. (F-J) Density effects on rosette leaf length of the 3 rd leaves, measured 3 weeks after sowing. (K-O) Density effects on flowering stem length, measured after bolting ( $\sim 6$ weeks after sowing). (P-T) Density effects on silique number, determined after bolting ( $\sim 6$ weeks after sowing). Seeds $(5,10$, 20, 40, and 60 per pot) were sown and grown at $24^{\circ} \mathrm{C}$ under LL. Data are presented as the means \pm SE $(n=10)$. Asterisks indicate significant differences, compared with the WT (Student's $t$-test, $p<0.05$ ).

Statistical analysis of gai and elf3 strains allowed us to identify their roles in flowering time, stem/leaf lengths, and silique number as a result of density effects. Cross- talk between the signaling pathways for GA and BR (Li and He 2013), BR and light (Lau and Deng 2010; Wang et al. 2012), and light and GA (Lor and Olszewski 2015) 

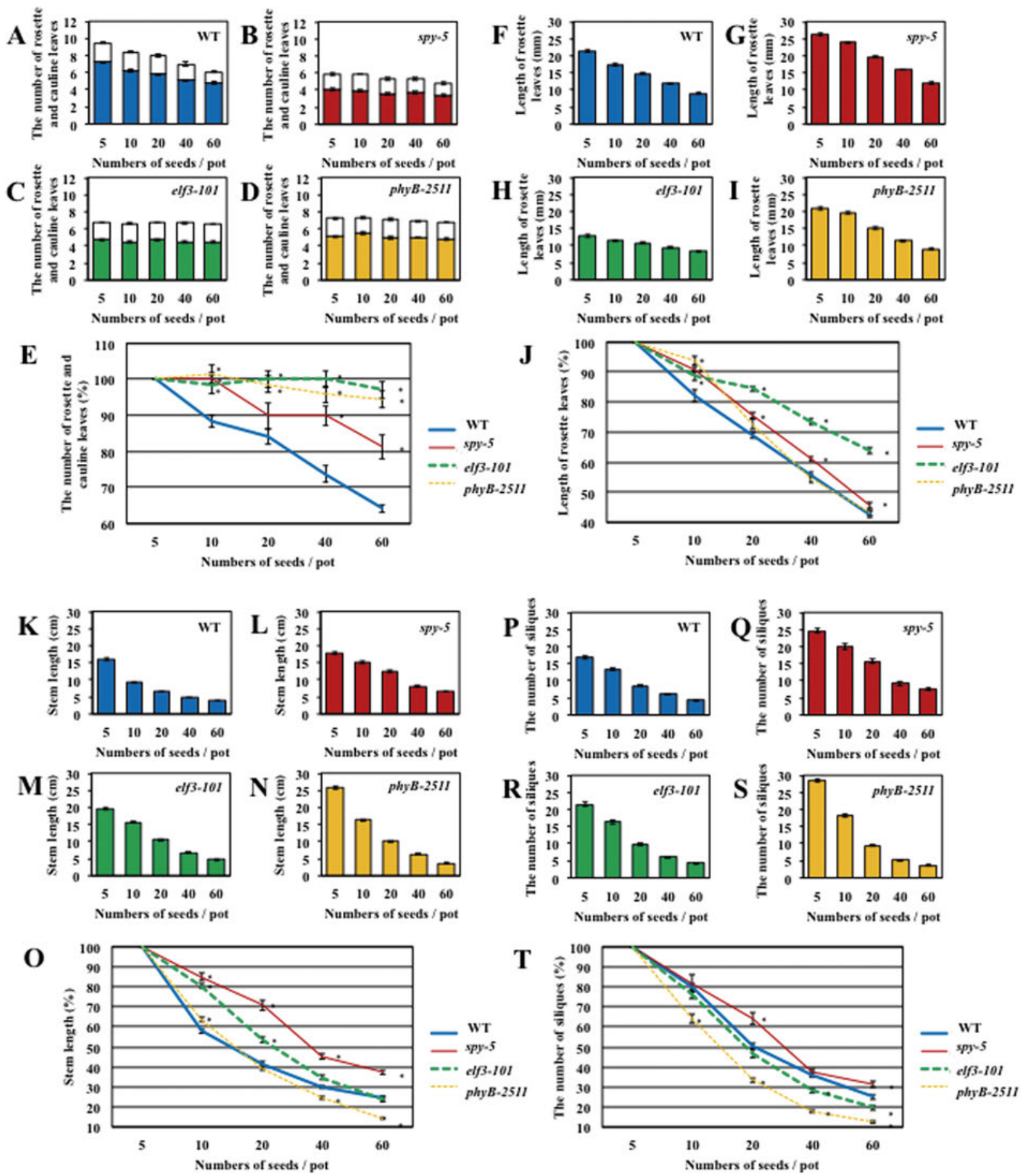

Figure 4. Density effects on flowering time, rosette leaf and flowering stem length, and silique number in $s p y$, elf3, and phyB mutants. Measurements are shown for WT (Ler, blue; A, F, K, P), spy-5 (yellow; B, G, L, Q), elf3-101 (red; C, H, M, R), and phyB-2511 (green; D, I, N, S) plants. Shown are the decreases in rosette and cauline leaf number $(\mathrm{E})$, rosette leaf length $(\mathrm{J})$, flowering stem length $(\mathrm{O})$, and silique number $(\mathrm{T})$ relative to those at densities of 5 seeds per pot. (A-E) Density effects on flowering time. Rosette and cauline leaves numbers were determined after bolting. (F-J) Density effects on rosette leaf length of the 3rd leaves, measured 3 weeks after sowing. (K-O) Density effects on flowering stem length, measured after bolting $(\sim 6$ weeks after sowing). (P-T) Density effects on silique number, determined after bolting ( $\sim 6$ weeks after sowing). Seeds $(5,10,20,40$, and 60 per pot) were sown and grown at $24^{\circ} \mathrm{C}$ under LL. Data are presented as the means \pm SE $(n=10)$. Asterisks indicate significant differences, compared with the WT (Student's $t$-test, $p<0.05)$.

have been reported. Common factors of the GA, BR, and light signaling pathways, including phytochromeinteracting factors (PIFs; de Lukas and Prat 2014), may play key roles in the control of flowering time, stem/leaf length, and silique number at high densities. How gai and spy mutations had similar effects at high densities, despite their opposite effects on GA signaling, remains unknown. Thus, investigation into the roles of other 
photoreceptors, including PHYA, PHYC, PHYD, PHYE, CRY1, and CRY2, on density effects is also warranted.

Flowering time of the early flowering mutants, elf3 and $p h y B$, was not accelerated at high densities; however, this may be due to an increased florigen activity in those mutants. Therefore, flowering times should also be tested in ELF3- or PHYB-overexpressing strains. The dwarf phenotype under extremely high-densities could be due to the lack of specific nutrients (Harper 1977; Willey and Heath 1961). Understanding such factors is necessary to detail the molecular mechanisms underlying density effects.

\section{Acknowledgements}

This study was supported in part by the joint research programs "Plant Transgenic Research Design, University of Tsukuba" of the MEXT (to T.M.), the joint research program implemented at the Institute of Plant Science and Resources, Okayama University, of the MEXT (to T.M.), grants-in-aid for Scientific Research on Priority Areas (MEXT; Grant number 23012037 to T.M.) and grants-in-aid for Scientific Research (C) (MEXT; Grant number 25440141 to T.M.).

\section{References}

Aihara T (1968) Competitive ability in mutant lines of a rice variety. Japan J Breed 18: 72-74

Busov VB, Brunner AM, Strauss SH (2008) Genes for control of plant stature and form. New Phytol 177: 589-607

Choe S, Dilkes BP, Fujioka S, Takatsuto S, Sakurai A, Feldmann KA (1998) The DWF4 gene of Arabidopsis encodes a cytochrome P450 that mediates multiple 22alpha-hydroxylation steps in brassinosteroid biosynthesis. Plant Cell 10: 231-243

de Lucas M, Prat S (2014) PIFs get BRright: PHYTOCHROME INTERACTING FACTORs as integrators of light and hormonal signals. New Phytol 202: 1126-1141

Fishman MA (1997) Density effects in population growth: An exploration. Biosystems 40: 219-236

Fujiwara S, Oda A, Yoshida R, Niinuma K, Miyata K, Tomozoe Y, Tajima T, Nakagawa M, Hayashi K, Coupland G, et al. (2008) Circadian clock proteins LHY and CCA1 regulate SVP protein accumulation to control flowering in Arabidopsis. Plant Cell 20: 2960-2971

Hardtke CS, Dorcey E, Osmont KS, Sibout R (2007) Phytohormone collaboration: zooming in on auxin-brassinosteroid interactions. Trends Cell Biol 17: 485-492

Harper JL (1977) Population biology of plant. Academic Press.

Jacobsen SE, Binkowski KA, Olszewski NE (1996) SPINDLY, a tetratricopeptide repeat protein involved in gibberellin signal transduction in Arabidopsis. Proc Natl Acad Sci USA 93: 9292-9296

Jennings PR, de Jesus J (1968) Studies on competition in rice. I. competition in mixtures of varieties. Evolution 22: 119-124

Kim GT, Tsukaya H, Uchimiya H (1998) The ROTUNDIFOLIA3 gene of Arabidopsis thaliana encodes a new member of the cytochrome P-450 family that is required for the regulated polar elongation of leaf cells. Genes Dev 12: 2381-2391

Kim GT, Fujioka S, Kozuka T, Tax FE, Takatsuto S, Yoshida S, Tsukaya H (2005) CYP90C1 and CYP90D1 are involved in different steps in the brassinosteroid biosynthesis pathway in Arabidopsis thaliana. Plant J 41: 710-721

Kira T, Ogawa H, Shinozaki K (1953) Intraspecific competition among higher plants. I. Competition-density yield interrelationships in regularly dispersed populations. J Inst Polytech Osaka City Univ D4: 1-16

Koornneef M, Hanhart CJ, van der Veen JH (1991) A genetic and physiological analysis of late flowering mutants in Arabidopsis thaliana. Mol Gen Genet 229: 57-66

Lau OS, Deng XW (2010) Plant hormone signaling lightens up: Integrators of light and hormones. Curr Opin Plant Biol 13: 571-577

Levin DA, Wilson JB (1978) The genetic implication of ecological adaptation in plants. In: Freysen AHJ, Woldendrop JW (eds) Structure and function of plant populations, North Holland Publ Co, Amsterdam, pp 75-100

Li QF, He JX (2013) Mechanisms of signaling crosstalk between brassinosteroids and gibberellins. Plant Signal Behav 8: e24686

Lor VS, Olszewski NE (2015) GA signalling and cross-talk with other signalling pathways. Essays Biochem 58: 49-60

Mizoguchi T, Wheatley K, Hanzawa Y, Wright L, Mizoguchi M, Song HR, Carré IA, Coupland G (2002) LHY and CCA1 are partially redundant genes required to maintain circadian rhythms in Arabidopsis. Dev Cell 2: 629-641

Mutasa-Göttgens E, Hedden P (2009) Gibberellin as a factor in floral regulatory networks. J Exp Bot 60: 1979-1989

Pacala SW, Weiner J (1991) Effects of competitive asymmetry on a local density model of plant interference. J Theor Biol 149: 165-179

Pearl R, Parker SL (1922) On the influence of density of population upon the rate of reproduction in Drosophila. Proc Natl Acad Sci USA 8: 212-219

Peng J, Carol P, Richards DE, King KE, Cowling RJ, Murphy GP, Harberd NP (1997) The Arabidopsis GAI gene defines a signaling pathway that negatively regulates gibberellin responses. Genes Dev 11: 3194-3205

Reed JW, Nagpal P, Poole DS, Furuya M, Chory J (1993) Mutations in the gene for the red/far-red light receptor phytochrome B alter cell elongation and physiological responses throughout Arabidopsis development. Plant Cell 5: 147-157

Suzuki S, Miyata K, Hara M, Niinuma K, Tsukaya H, Takase M, Hayama R, Mizoguchi T (2016) A loss-of-function mutation in the DWARF4/PETANKO5 gene enhances the late-flowering and semi-dwarf phenotypes of the Arabidopsis clock mutant lhy-12;cca1-101 under continuous light without affecting FLC expression. Plant Biotechnol, in press

Wang ZY, Bai MY, Oh E, Zhu JY (2012) Brassinosteroid signaling network and regulation of photomorphogenesis. Annu Rev Genet 46: 701-724

Weiner J, Freckleton RP (2010) Constant final yield. Annu Rev Ecol Evol Syst 41: 173-192

Willey RW, Heath SB (1961) The quantitative relationships between plant population and yield. Adv Agron 21: 281-321

Yamamoto K, Takahashi K, Hara M, Miyata K, Hayama R, Mizoguchi T (2016) Density effects on late flowering mutants of Arabidopsis thaliana under continuous light. Plant Biotechnol, in press

Zagotta MT, Hicks KA, Jacobs CI, Young JC, Hangarter RP, Meeks-Wagner DR (1996) The Arabidopsis ELF3 gene regulates vegetative photomorphogenesis and the photoperiodic induction of flowering. Plant J 10: 691-702 